\title{
Arbor
}

\section{Des châteaux, des cités, des hommes et des livres}

\section{Pierre Naudin}

Arbor CLXX, 671-672 (Noviembre-Diciembre 2001), 559-569 pp.

Je devais avoir six ans lorsque je fus mis en présence dun témoin du Moyen Age. Mes parents, qui étaient pauvres, avaient eu, je ne sais comment, loccasion de se rendre á Dieppe un dimanche. Nous avons quitté Choisy-le-Roi, notre ville, au lever du soleil pour monter, á Paris, dans un train dont le roulement monotone mendormit aussítôt. Sous une pluie continuelle, le chemin de fer nous conduisít dans cette cité portuaire sans autre vestige du temps passé que son château proche de la mer.

Demblé, cette énormité de pierre grise coiffée dardoise me parut sinistre. Sa visite confirma mon impression. Jen sortis lâme et le corps glacés. Il me tarda de repartir. La journée me parut trés longue.

Jignorais tout, alors, de notre Histoire. Je ne fus guére enclin á en savoir davantage. Pourtant, je finis par my intéresser: les gravures de mes livres scolaires mimportérent cependant plus que les textes.

Mon second contact fut différent de celui de Dieppe. Aprés avoir thésaurisé toute leur existence, mes grands-parents achétèrent, en 1934, une maison á Briatexte. Ce village situé a égale proximité de Toulouse et dAlbi avait été fondé, sous Philippe le Bel, par son vassal, Simon Briseteste, que le roi de France avait nommé, le 27 avril 1287, sénéchal de Carcassonne. Cet important serviteur de la Couronne semble avoir été un homme honnête et consciencieux. Aprés une visite aux berges du Dadou, la rivière qui coulait á proximité des ruines de lancien château de Thoëls, il décida de creer ce quon appelle toujours une bastide. Dès 1289, des maisons furent bâties et lon entoura léglise dune place á arcades, sorte de cloître civil á usage commercial appelé place des couverts. 
Contrairement à la mélancolie que j'avais éprouvée dans mon enfance prime à la vue dun château blafard et inhospitalier, je reasentis devant cette maison de lAncien Temps en partie délabrée, une sorte débahissement rehaussé dun singulier plaisir: elle me donnait confiance. Pourtant, sa façade dotée dun encorbellement était rébarbative! Pendant mes trois mois de vacances annuels, elle allait devenir mon refuge. De ma chambre, avant que le sommeil me prît, jécouterais le bruiesement dune rivière qui hélas! souillée par les rejets des mégisseríes de Graulhet, une cité voisine, est devenue la plus polluée de France.

Mes grands-parents avaient chargé mon pére de remettre leur second foyer en état puisquils y finiraient leur vie. Il disposaint de tout son temps, étant pensionné de la guerre de 1914-18. Il sy consacra tandis que nous étions logés chez des parents de ma grand-mére qui avait passé toute sa jeunease á Briatexte.

Cette maíson était lune des plus anciennes du village. Son agencement me parait encore un modele dont certaina architectes pourraient sinspirer. Tandis que mon pére et les maçons quil avait sollicités consolidaient le rez-de-chauasée, une partie de celui-ci seffondra: la colonne de sóutenement, en briques et mortier, fut engloutie dans une cavité qui, sans aucun doute, etait un machicoulís aménagé dans les anciennes murailles. Jajoute quau centre de la cave, une profonde fosse pareille á un entonnoir renversé avait été creusée. En 1950, mon épouse et moi avons voulu la sonder. Nous avons retiré des pelletèes de détritus de cuir de toutes formes: avant son acquisition par mes grands-parents, la maison avait été occupée par un cordonnier. Que contenait cette fosse? Etait-elle lentrée dun souterrain? Je le crois mais ne puis en apporter la preuve: ma cousine reçut cette maison en donation partage. Elle sen défit immédiatement. Bien que le prix de vente eût été des plus modeste, je ne disposais pas des fonds pour lacquérir.

\section{Le troisiéme contact}

Mon troisième contact avec le Moyen Age date de 1936. Alors que nous étions en vacances, mon père memmena á Cordes, á $2 \mathrm{O}$ kilométres de Gaillac. Environ 100 kilomitres, á bicyclette (aller-retour).

Cordes est désormais célèbre: un peintre médiocre la baptisée Cordes sur-Ciel, formule banale dont se délectent les gens du pays. Cette très ancienne cité a conservé maintes maisons et monuments dépoque. On ne peut mieux la comparer quá un Mont-Saint-Michel terrestre.

Cette pyramide de joyaux architecturaux. existait avant mame que Simon de Montfort, (1150-1218) eút conquis le Languedoc. Lorsque 
Des châteaux, des cités, des hommes et des livres

nous la visítâmes, les rues étaient «en escaliers». Les maisons dont les propritaires etaient morts pendant une épidémie (la peste ?) et dont les entrées etaient closes depuis des siécles, exhibaient sur leurs portes de grandes croix vermeilles. Maintes autres, dans cette cité moribonde, étaient á vendre.

Je me souviens davoir erré sans trop de curiosité dans cette bastide. Il y avait plus de chiens que de gens dans les rues. Bien quelle eût souffert de la guerre qui avait opposé les guerriers du Nord et du Sud, je ny percevais rien de tragique. Je fus déçu.

Je le fus plus encore en 1968 lorsque jy revins. La cité renaissait. J-eusse dû men réjouir mais, comme le Mont-Saint-Michel, elle était livrée au mercantilisme. Elle ne vivait, elle aussi, que du touriame.Je ny reviendrai pas.

\section{Le quatrieme contact}

Mon quatrième contact fut plus intéreasant.

Alors que mon épouse, ma fille et moi passions nos vacances en Normandie, dans le Cotentin et plus précisément á 6 kilométres de Coutances, nous découvrîmes, lors dune promenade, un château ruiné enveloppe de líerre. Cet inmense vestige du passé engoncé dans sa parure verdoyante développa sous nos regards, au fur et á mesure que nous le visitions, des beautés qui, cette fois, me touchérent. Il existait encore une porte, des boiseries. Je pouvais mentalement reconstituer la totalité de cette ruine. Il y avait même une tour intacte: la Tour de la Fée. Elle était vêtue de plantes parasites mais je pouvais imaginer sans peine la dame de jadis qui lavait hantée, ainsi que les seigneurs et les serviteurs sadonnant á leurs tâches quotidiennes. Le nom de ce château, Gratot, simprima dans mes pensées dune façon indélébile ainsi que celui de la famille qui y avait vécu: les Argouges.

Personne, alors, ne visitait Gratot. Il était pourtant proche dune route dou on le voyait, majestueux, esseulé, abandonné impardonnablement á une végétation dévorante. Nous y allâmes cinq ou six fois en un mois et pendant quelques années, sitôt installés dans la maison de campagne oú nous vivions loin des servitudes parisiennes, notre première visite ètait pour ce château.

Nous fûmes satísfaits lorsque des jeunes commencérent á le restaurer. Le lierre dísparut et je maperÇus que ce qui avait fait la beauté de cette forteresse entourée dune douve oú elle se mirait, cátait la profusion de sa verdure et sa solitude. 
De savantes et nécessaires réfections avaient accompagné cette opération de nettoyage. Au fur et á mesure quelle setaient effectuées, Gratot avait perdu son âme. Il nétait plus question pour moi, devant ou dans ce aquelette de pierre, dimaginer quoi que ce fût. Il y avait quelques touristes. Ils se photographaient parmi les pans de murs, auprés dun contrefort, sur le seuil düne voúte sombre. Gratot nétait plus quun immense-ossuaire. Jai vu dés visiteurs séloigner lesprit aussi triste quinsatisfait.

Un jour oú ma déception se révélait plus grande quá lordinaire, je décidai par lécriture de ressusciter ce château; de lui rendre son aspect ancien et sa population. Cependant, jhésitai. Pour mélancer dans mon entreprise, il me fallait tout apprendre de la vie, des moeurs, des événements survenus lors des années 1300 á 1400. Je mis cinq ans á me documenter sans ácrire une ligne de cet ouvrage, mais en usant mes plumes á prendre des notes dans nos deux grandes bibliothèques: la Nationale et l'Arsenal. Et je nosai toujours pas écrire la premiére ligne de mon récit.

Pourquoi? Eh bien, parce quá mes debuts en littérature, javais été bloqué par une réflexion de lhomme quí avait accepté avec enthousiasme les quatre feuillets dun conte que javais envoyé au journal L'Aurore sans grand espoir d'etre publié dans la page quotidienne consacrée a la vie littéraire. Il se nommait Albert-Jean. Chaque mois, il me publiait un conte, cest-á-dire une courte histoire en 4 pages manuscrites. Un jour oú, dans le «corset« qui nous était imposé, á une vingtaines dauteurs et á moi, je décrivis le retour dun croisé en son château. Mon héros sétait absenté de longues années. Si longtemps que son épouse, le croyant mort, sétait remariée. Une joute devait départager les deux maris.

Jlavais été content de cette histoire. Elle nétait point originale et je savais que des remariages de cette espéce avaient existé. Or, je reçus du directeur littéraire de L'Aurote une lettre que je nai pas conservée mais oú il me disait en substance: "Renoncez á écrire sur le Moyen Age. Ce nest pas un sujet pour vous».

Ce jugement mobsédait.Jattendis un autre événement pour matteler ou non á la besogne.

\section{Une rencontre décisive}

Je doía ma rencontre décisive á lEspagne. Jy vins pour la premiére fois en 1971 et la traversée du pays, pour me rendre á Castellón de la Plana, fut un enchantement. Cette année-lá, je découvris Peñiscola, 
Des châteaux, des cités, des hommes et des livres

et pendant plus de dix ans, cette cité fut pour ma famille et moi un lieu idéal de vacances.

Evideament, la forterease avait tout ce quil fallait pour me séduire. Toutefois, elle ne me suffisait pas. Nous découvrimes, sur la grand-route de Tarragone á Valence, des châteaux perchés sur des montagnettes. Il me fallut les approcher. En pleine chaleur, un jour, nous décidámes dune escalade pour visiter lun deux. Il dominait le village de Santa Magdalana de Pulpe.

Ce fut une ascension si p'enible dans les rochers et les pierres que notre caniche y voulut renoncer. Je le portai. En haut, je fus tout dabord déçu. Le monument qui, den bas, mavaít semblé preservé des ans et des intempéries, nétait quune ruine oú certains murs subaistaient encore, aussi hauts quá leur origine. Quelques instants plus tard, une sorte denchantement agit sur moi, que je navais jamais encore éprouvé dans les châteaux «français», surtout ceux du Languedoc.

Comment dire? Il me semblait que cette forteresse qui peut-être navait jamais reçu de visite depuis des décennies, mouvrait son coeur. Cela peut paraître insensé de le révéler, mais je pus imaginer enfin. dans leur complétude étrange et colorée les hommes et les femmes qui avaient vécu dans ce castillo perdu, livré aux assauts des vents et des pluies venus de la mer ou des montagnes. Les souffles chauds mapportaient leur haleine et il sen fallait de peu que jentendisse leurs propos, leurs rires et le bruit des sabots des chevaux et des mulets sur la pierraille dun chemin qui nexistait plus.

Lenvie de décrire lEspagne du XIVe siécle sajouta su désir de créer des personnages qui fussent authentiques ou qui en eussent vraiment lapparence. lls devraient voir ce que je voyais, entendre ce que jentendais éprouver en certaina lieux ce que jéprouvais.

Il me restait á faire quelques rencontres édifiantes. Ce furent, les années sajoutant aux précédentes, Guadamur, Alarcon et Toléde.

Le premier château reçut deux fois ma visite. Le second trois foia. Jai admiré la beauté sauvage, lunaire, dun site oú je ne me suis pas contenté derrer a proximité de la citadelle. Jen ai escaladé les escarpements pour aller rôder á lentour des tourelles de guet et, lá encore, imaginer -ayec moins dacuité quau "castillo perdu»- les personnages dautrefois.

Lultime poussée qui me fut donnée vers lécriture dune fresque historique (et jen suis bientôt á son $17 \mathrm{eme}$ volume consacré á la Guerre de Cent Ans en France, en Angleterre et en Espagne, dont 3, provisoirement á cette dernieré), Iultime poussée porte un nom précis: Toledo.

Je fus doublement enthousiasmé par cette cité fantastíquement belle et par un homme qui, en lannée 1972, forgeait des armures que 
jamais je navais vues daussi prés. Cet armurier, cest Félix del Valle, et je dois dire, car cest la vérité, que sans mon long arrêt dans son atelier, sans ses propos sur son métier, - je devrais dire sur ses oeuvres-, je naurais pas aussi complétement meublé ma cervelle avec les éléments casentiels de lexistence au Moyen Age: les armes, les armures, les chevaliers, leurs amours et leurs haines. Je pouvais donc habiller de fer mes châtelains, décrire leurs passions et leurs jeux -sans oublier leurs guerres. Jajoute que pour compléter mon éducation sur ces hommes de fer, je suis allé trois fois á Segovia dans lintention de visiter lentement lAlcazar. Ce fut toujours un éblouissement.

\section{Lultime rencontre}

La derniere rencontre qui paracheva mon éducation porte un nom prestigíeux: Carcassonne. Cette vaste cité. pourvue dune double enceinte et dont certaines murailles et parties de quelques tours sont d'origine gallo-romaine, fut sauvée par un homme extraordinaire, doué dune puiasance de travail phénomenale: Eugène-Emmanuel Viollet-le-Duc (1814-1879). Grand admirateur de larchítecture du Moyen Age, il avait voyagé a travers la France en compagnie de son ami, Prosper Mérimée, alors inspecteur des Monuments historiques, écrivain et auteur dun livre remarquable sur Pedro le Cruel.

LEmpereur Napoléon III épousait les idées des deux hommes quant á la protection des monumento érigés en une lointaine époque et dont les générations et les gouvernements antérieurs au Second Empire sétaient désintéressés. Disposant des fonds considérables alloués par lEmpereur et ses ministres, Viollet-le-Duc avait restauré,avant de se pencher sur les décombres de Carcassonne, la basilique de Vézelay, les églises Saint-Germain-des-Prés et Saint-Sernin de Tolouse, et Notre-Dame de Paris. Il allait plus tard reconstituer le chateau de Pierrefonds en sinspirant toujours des formules stylistiques médiévales. Génie de larchitecture, il fut parfois critiqué. Son oeuvre écríte, ses dessins, ses gouaches et ses tableaux sont encore une source de références pour qui veut avoir une vision non pas approximative, mais juste du Moyen Age.

Il convient, pour visiter Carcassonne, de se lever á laube et de se presenter seul devant la Porte Narbonnaise, lentrée principale de la cité, afin dy errer á son gré sans que des touristes ne viennent ni gêner les regards ni souiller les méditations.

Une fois le pont-levis franchi, il importe de négliger les lices sises entre les deux enceintes et de cheminer dans létroite rue qui méne 
Des châteaux, des cités, des hommes et des livres

au château comtal dont lentrée, flanquée de deux tours portieres, defend lintimité dun jardín. Pendant la guerre du Nord contre le Sud, Simon de Montfort y avait installé sa demeure.

Une fois le seuil entre les tours, on découvre le château que jouxte une maison récente, á colombages, réplique de celle, trés ancienne, que lon peut admirer sur la place de la cité dAlet-les-Bains, á 25 kilomètres de Carcassonne. Le château visité, la basilique Saint-Nazaire et Saint-Celse offre á tous les amateurs dart roman et gothique qui veulent errer dans les draperies impondérables répandues par les vitraux, des trésors multiples essentiellement du XIVe siécle. Ils flattent de proche en proche les yeux, lesquels peuvent se reposer sur les ornements et déchiquetures de pierre oú les sculpteurs de jadis donnérent cours á cette imagination et ce goût du fantastique qui font le charme des vieilles églises et des cathédrales.

Aprés cette cure de sérénité sous de hautes voûtes fraîches, il nereste plus qua errer dans la cité. Il convient dy marcher su hasard et de ne pas trop attarder ses regards sur les maisons et les boutiques puisquil ny a, en vérité, plus rien de médieval á considérer.

Et cest bien le paradoxe de cette citadelle aux murailles formidables et aux abords tentateuts. Excepté quelques façades oú lon discerne encore linfluence de Viollet-le-Duc, les dites maisons sont des plus ordinaires. Comme á Cordes et au Mont-Saint-Michel, le mercantilisme régne. Il y a pléthore de restaurants, de boutiques consacrées á la vente des glaces, gâteaux, sandwiches: tout ce qui peut se manger en marchant, et les rues sont jonchées de détritus. Les boutiques de souvenirs abondent et ceux-ci sont du plus mauvais gôut. Leurs présentoirs ne séduisent que les enfants. Deux exceptions cependant: dans le magasin de M.Aleasandri, place Saint-Jean, sont exposées des armes et des tapisseries de qualité. A lechoppe Damsel, dans la ruelle SaintSernin accedant á la place Saint-Marcou, une Anglaise vend des objets de son pays. Ils méritent plus quun regard.

\section{Quand le modernisme prejudicie l'Ancien}

Un an avant dentrer dans le nouveau siècle, des boutiques modernes furent crées place Saint-Jean, assez éloignées, heureusement, de celle de M. Alessandri. Elles sont autant dinsultes á larchitecture medievale et contribuent à la dégradation dune cité qui devient «invisitable» á partir de 9 heures jusqua 19 heures. Une foule immense, souvent sale, fréquemment índécente, sy coagule non point pour admirer quelques beaux vestiges mais pour sy gaver de tout ce qui peut se manger. 
On se demande pourquoi tant de Béotiens, de mendiants, de visiteurs inutiles se déplacent á Carcassonne puisquils ny contemplent rien et néprouvent rien dautre que les démangeaisons de leur estomac et de leur gorge fréquemment asséchée. Il y a mieux encore au chapitre de la déception: les guides chargés de la visite des remparts sont trop peu nombreux, lété, pour piloter les rares touristes soucieux de sinstruire. De jeunes stagiaires sont embauchées. Un leur a fait préalablement la leçon, et sans doute certains vieux guides malicieux ont-ils glissé dans leurs propos quelques sornettes quelles sempressent dintroduire naïvement dans leurs commentaires. Je ne résiste pas á lenvie de conter lanecdote suivante, non seulement parce quelle est authentique, mais aussi parce quelle révèle un laisser-aller singulier.

En 1995, sachant que javais «fabriqué« moí-même et pour mon compte un équipement militaire du Xlll siécle, un général ami me demanda de participer avec mon épouse à une fête médiévale quil organisait á Mazéres, á environ 50 kilométres de Carcassonne. Il voulait, á tout prix, que dans le défilé, il y eut un chevalier et sa dame á vrai dire authentiques.

Mon haubergeon était composé de 60.000 anneaux et mon camail de 11.000 .

Une semaine avant de participer á cette fête, ma femme et moi décidâmes daller errer dans Carcassonne. Une visite allait avoir lieu. Il y avait, entourant une jeune cicerone, une quinzaine de personnes. La curiosité nous prit, et bien que connaissant la cité en long et en large, nous nous joignimes a elles.

Demblée, je relevai maintes erreurs dans le discours de la jeune fille. Je minterdis, dy apporter la moindre modifications, mais je souffrais de mon silence.

Nous arrivámes á la tour dite de llnquisition, á la sortie de laquelle séleve un escalier dune quarantaine de marches. Au pied de celui-ci, la jeune commentatrice nous tínt ce propos:

-Mesaieurs - dames, pour monter en haut de cet escalier.les soldats étaient obligés d'ôter leur cotte de mailles.

Je contestai aussitôt cette sorttise énorme. La jouvencelle me considéra comme si etais un imbécile qui ne connaissait rien á l'affaire et mexpliqua quune cotte de mailles était lourde (j), rigide (i!) et conclut quelle avait raison.

Je mabstinas de poursuivre un dialogue qui nous eût courroucés lun lautre.

\section{Il convient de démontrer}

En revenant á Alet-les-Bains, notre lieu de résidence, je dis á mon épouse:

- Nous y retournerons demain. Il faut que je donne une leçon á cette péronnelle. 
- Tu vas te faire remarquer, me reprocha-t-elle.

Le lendemain, ma cotte de mailles et mon camail dans un sac en plastique - javais renoncé á mon épée-,nous revînmes á Carcassonne. Par miracle, notre jeune guide allait partir en tournée. Nous reconnut-elle ? Je-ne le crois pas.

Nous nous joignîmes aux visiteurs et entendîmes les mêmes absurdités que la veille. Parvenus á la Tour de llnquisition, je dus subir le même inepte commentaire sur la montée de lescalier.

- Permettez, dis-je alors á la pimprenelle.

Jouvris mon sac, en tirai mon camail $(1,200 \mathrm{Kg})$ et mon haubergeon (environ $18 \mathrm{~kg}$ ). Et je madressai aux touristes:

- Vous venez dentendre, messieurs et mesdames, une explication déraisonnable. Quelqun dentre-vous veut-il endoaser cette cotte et se coiffer de ce camail ?

Quittant sa fiancée,un jeune homme accepta mon offre.--;Je laldai á endosser mon habit de fer et á se coiffer du camail.

Il étaít au bas des marches.

- Montez-les en courant, lui dis-je, comme si nous étions attaqués.

Il gravit les marches aisément á la course. Quand il fut au sommet, je me tournai vers notre cicérone.

- Pardonnez-moi, lui dis-je, mais quand on veut sexprimer sur le Moyen Age, il convient de se documentar.

Puis je mexcusai de lavoir humiliée.

Elle ne protesta pas. Je sentis, évideament, sa colére gliaser de ma tête á mes pieds tandis que je remettais dans mon sac mes deux vétements de fer. Les touristes ne souriaient point. Je suis sûr que certaines femmes men voulálient davoir troublé un jeune esprit dont je nadmettais pas quon eût versé dedans quelques sottises. En fait, javais fourni une leçon á tous. Jusqua la fin de la visite, je me sentis parfaitement tranquille. Jagis toujours avec mes semblables non pas selon ce quils sont, mais selon ce quils devraient être. Cest une forme de générosité que de les instruire même si la vérité peut les indisponer a mon égard.

\section{Et, puisquil faut conclure....}

Revenons aux châteaux et cités concernant les bonnes leçons qulils me fournirent. 
Le village occitan de Briatexte, qui fut sí beau dans ma prime jeunesse le demeura, je croia, jusquen 1980. Un jour, allant, comme chaque année, me recueillir sur la tombe de mes grands-parents, je rencontrai le maire. Aprés les propos dusage, il mannonça fièrement:

- Nous avons modernisé la place des couverts. Allez-y! ... Vous pourrez admirer le changement.

Nous y allâmes, mon épouse et moi,et fûmes scandalisés. La belle place au centre de laquelle, cent ans plus tôt, on avait détruit une église qui avait été construite lors de la création de la bastide démolie en 1330, reconstruite et qui existait encore au XVIIIe siéclevavant de disparaitre á jamais, la belle place, dis-je, avait perdu tout son caractare ancestral. On lavait rehauasée et dallée. Des lampadaires 1900 et une fontaine anachronique (de fort mauvais goût) «juraient« avec les bâtiments du pourtour. Cétait un affreux blaspheme asséné à un ouvrage antique, une irreparable gangréne inoculée á des témoins de pierre qui pouvaient attester de la vieillease de Briatexte.

Mieux encore: une espéce de muret séparait les premiéres maisons de la route, bien quil ny eût jamais eu daccident en cet endroit, Léclairage consistait en des lampadaires qui se voulaient modernes et qui sont affreux! Lorsque nous repassámes, en 1999, on détruisait des maisons centenaires comme lon avait détruit le vieux lavoir pittoresque pour le remplacer par un parking!

Je ne retournai pas rue du Moulin pour contemplar mélancoliquement la maison de mes grands-parents, ni même au vieux moulin dont de nouveaux propriétaires avaient détruit tout le charme. Jajoute quun viaduc surplombait le Dadou. Sa destruction inutile avait fait deux morts .Nous partimes, mon épouse et moi, comme on fuit.

Je ne dirai ríen de Gratot qui tient pourtant une place énorme dans mes récits. Il est sinistre. A quoi bon sy arrêter encore.

Javais connu Peñiscola en 1971, alors que le château et le petit víllage blanc niché contre lui, composaient un site extraordinaire. Progreasivement., des hôtels sy sont bâtis partout, anarchiquement, rendant ce site insupportable á ma vue. Les montagnes á lentour ont, été assaillies et devorées par des maísons, des maisons et encore des maisons: cest un tohu-bohu auquel se joint, si jose dire, le tintamarre des annonceurs célébrant les qualités contestables des restaurants et des établissements nocturnes. Ce nest pourtant pas á regret que cette cité figurera bientôt dans un des tomes de ma troisiéme fresque puisque Benedict XIII (Papa Luna) y joue un rôle important.

Je pense quAlarcon et Toléde sont inchangés. Toute modernité, dans les antiques cités, me paraît sacrilège. Cest pourquoi, si je vais á 
Des châteaux, des cités, des hommes et des livres

Carcassonne, ce nest pas pour admirer une antiquité qui, de lintérieur, hormis quelques monuments préservés, ne mapporte sentimentalement et historiquement rien. Cest pour y rencontrer quelques amis: léditeur Loubatiéres dont les deux librairies sont des lieux de silence et de quíétude et les deux personnes que jai citées plus haut. Lorsque ma vue effleure le château comtal, je me souviens du temps oú lon avait eu lexcellente idée dy installer un musée du costume consacré au XVe siécle. Cétait une exposition splendide, les personnages et les vêtements ayant été créés par Bermans, de Londres. On eût dit les femmes et les hommes vivants.

Eh bien, ils furent vendus, je dirai même bradés á un cafetier qui en fit une exposition décevante. Alors que, prédédemment, les sujets en fibre de verre «évoluaient» dans un cadre adéquat, ils furent transférés dans une maison ordinaire oú, du même coup, ils perdirent toute leur distinction.

Par quoi les a-t-on remplacés ? Par les fanfaronnades de lart dit «contemporain" dont on sait, évidemment, quil est la négation du beau et de lutile.

La conclusion de ce bref voyage dans lAncien Temps ne saurait être optimiste. Si le Passé nous a fait ce que nous sommes, il convient de le respecter. Toute atteinte su patrimoine dont nous sommes les dépositaires est un crime qui, hélas! ne peut être puni. Il ny a plus guére de respect pour les vieilles pierres, mais une certitude me console: toutes les innovations et constructions parasitaires dureront moins longtemps que leurs «ancêtres». Qú elles se ruineront delles-mêmes ou des pelleteuses et des marteauxpiqueurs, aprés quon eut repéré des lézardes dans leurs murs permettront de les remplaceront par des créations plus laides encore ... si la chose est possible.

Cest ce qui me consterne...et me console. 